\title{
A Zero-field Nuclear Magnetic Resonance Spectroscopy Study on the Structure Property Relationship in Co thin Films
}

\author{
Patrizia Fritsch ( $\nabla$ p.fritsch@ifw-dresden.de) \\ Leibniz Institute for Solid State and Materials Research \\ Juriaan Lucassen \\ Eindhoven University of Technology
}

Henk Swagten

Eindhoven University of Technology

\section{Bernd Büchner}

Leibniz Institute for Solid State and Materials Research

\section{Reinoud Lavrijsen}

Eindhoven University of Technology

\section{Sabine Wurmehl}

Leibniz Institute for Solid State and Materials Research

\section{Research Article}

Keywords: Dzyaloshinskii-Moriya interaction (iDMI), magnetic , out-of-plane (oop), magnetic stiffness

Posted Date: December 8th, 2020

DOl: https://doi.org/10.21203/rs.3.rs-120487/v1

License: (9) (i) This work is licensed under a Creative Commons Attribution 4.0 International License. Read Full License 


\title{
A zero-field nuclear magnetic resonance spectroscopy study on the structure property relationship in Co thin films
}

\author{
P. Fritsch, ${ }^{1,2}$ J. Lucassen,${ }^{3}$ H. J. M. Swagten, ${ }^{3}$ B. Büchner, ${ }^{2}$ R. Lavrijsen, ${ }^{3}$ and S. Wurmehl ${ }^{2}$ \\ ${ }^{1}$ Corresponding author: p.fritsch@ifw-dresden.de \\ ${ }^{2}$ Leibniz Institute for Solid State and Materials Research IFW-Dresden, \\ Institute for Solid State Research, Helmholtzstraße 20, 01069 Dresden, Germany \\ ${ }^{3}$ Department of Applied Physics, Eindhoven University of Technology, \\ P.O. Box 513, 5600 MB Eindhoven, Netherlands
}

\begin{abstract}
The interfacial Dzyaloshinskii-Moriya interaction (iDMI) was investigated in Co thin-films with different combinations of seeding and capping layers in previous works. The variations in the local magnetic out-of-plane (oop) anisotropy $K$ as a function of film thickness $t$ lead to additional contributions that make the quantification of the iDMI more challenging. These additional contributions were specifically attributed to the transition of $f c c$ Co to hcp Co at a critical thickness $t_{\mathrm{cr}}$. In this work we clarify this issue by investigating the local structure and local magnetic stiffness and their contribution to the oop anisotropy $K$ in two $\mathrm{Pt} / \mathrm{Co}(t) / \mathrm{Ir}$ film stacks with $t=10 \mathrm{~nm}$ and $t=25 \mathrm{~nm}$. Zero-field nuclear magnetic resonance spectroscopy (ZF NMR) is the method of choice as it is a non-destructive and sensitive probe to examine structure and magnetic properties of nano-sized samples. We describe the evolution of the Co allotropes ( $f c c \& h c p)$ and stacking fault disorder with the increase of $t$ and illustrate the structure-property relationship between the local environments of Co and their respective magnetic stiffness.
\end{abstract}




\section{INTRODUCTION}

Interfacial Dzyaloshinskii-Moriya interaction (iDMI) is nowadays one of the hot topics in thin-film magnetism. Specifically, these asymmetrical magnetic exchange interactions are realized in thin films at symmetry breaking interfaces, e.g., between heavy metals with high spin-orbit coupling (SOC) and ferromagnetic materials typically from $3 d$ elements [1-5]. Besides being of fundamental interest, studies of iDMI are also of technological interest as they are considered to realize new magnetic phenomena like the formation of chiral domain wall (DW) dynamics and magnetic skyrmions with their promising application in future nanoelectronic devices $[3,6,7]$. Nanoscale thin films of elemental Co sandwiched between $5 d$ elements, e.g., Ir or Pt (both elements with high SOC) are the paradigm materials to study iDMI.

Metallic, elemental Co exists in three allotropes, body-centered cubic (bcc), face-centered cubic $(f c c)$, and hexagonal close packed $(h c p)$, the two latter phases are distinguished by the stacking order of their closest packed layer. Denoting the first Co closed packed layer as $A$ and the second layer $B$ on top of half of the gaps of the $A$ layer, there are two possible ways of arranging the next layers. Their assignment depends on the choice of the gap in the second layer $B$ on which the third adjacent layer is placed. Each packing variant yields a closed packed lattice, $f c c$ with $A B C A B C$ stacking, and hcp with $A B A B A B$ stacking. Specifically, $f c c$ has the $A B C A B C$ stacking in the [111] direction, while the hcp phase has an $A B A B A B$ stacking in the [0001] direction. Hcp is the thermodynamically stable phase at ambient conditions [8]. Since transition energies between those two allotropes are fairly low, both $f c c$ and $h c p$ may coexist under certain conditions[8, 9]. Both Co allotropes show different magnetic properties and magnetic anisotropies with implications for film stacks consisting of Co used to probe the iDMI.

Recently, Lucassen et al. examined the iDMI in Co film stacks with varying thicknesses (4 to $26 \mathrm{~nm}$ ) utilizing propagating spin-wave spectroscopy (PSWS)[10]. It turned out that for investigating the nonreciprocity as a function of Co layer thickness for $\mathrm{Pt} / \mathrm{Co} / \mathrm{Ir}, \mathrm{Pt} / \mathrm{Co} / \mathrm{Pt}$ and $\mathrm{Ir} / \mathrm{Co} / \mathrm{Pt}$ systems, a careful disentanglement of contributions other than the iDMI to the nonreciprocity is vital. The nonreciprocity is dramatically enhanced by a change in the Co modification, $f c c$ to $h c p$ at a critical thickness $t_{\text {cr }}$ of $12 \mathrm{~nm}$. Please note, that this chritical thickness is dependent on the underlying growth conditions. Connected to the 
phase transition between the two Co allotropes is also the rise of the oop anisotropy $K$ above the threshold $t_{\mathrm{cr}}$. Understanding the structural differences that in turn change the magnetic properties is therefore of prime importance.

Zero-field nuclear magnetic resonance spectroscopy (ZF NMR) allows to study phase compositions quantitatively and to investigate the local magnetic anisotropy using the optimal radio frequency $(\mathrm{RF})$ power for each specific local structural environment[11-13]. This method is also known as internal field NMR as it uses the electronic exchange interactions as a source for the nuclear Zeemann splitting and probes directly the hyperfine field around the nucleus of magnetically ordered materials without the use of an externally applied magnetic field (see Portis and Gossard [14]). NMR has been proven helpful to clarify structureproperty relationships in bulk Co[14-17], Co/X multilayers[18-21] and thin films[22-24] over the decades. This renders ZF NMR as a method of choice for the study at hand.

Part of the study, namely the ZF NMR frequency spectra and its superficial interpretation, was already published in the supplemental of Lucassen et al.[10]. In this work, we go beyond the previous study and present in detail the phase and defect composition in two Co film stacks with different thickness. This detailed analysis includes a rationalization of the ideal radio frequency $(\mathrm{RF})$ power of the NMR pulse and the frequency shift as an indicator of lattice strain. We show the defect and phase composition driven effect on the magnetic stiffness in nanoscale Co thin films by examining the optimal RF power as a measure for the locally and structurally resolved magnetic anisotropy.

\section{RESULTS \& DISCUSSION}

Two thin films of different thickness were measured according to the procedure described in section IV. The thickness $t$ of the films were chosen to be below $(10 \mathrm{~nm})$ and above $(25 \mathrm{~nm}) t_{\mathrm{cr}}$ of about $12 \mathrm{~nm}$ where the non-reciprocity is enhanced as reported by Lucassen et al. [10]. The NMR spectra in Fig. 1 (upper panels (a) and (b)) show the recorded and power-corrected spin-echo intensity of the probed ${ }^{59} \mathrm{Co}$ nuclei as function of frequency. Both spectra exhibit a set of partially overlapping resonance lines in the frequency range 200$240 \mathrm{MHz}$ but with different shares of the individual sub-lines. To further analyze the NMR data, we used different fitting models consisting of a sum of different numbers of Gaussian lines where each of those lines refers to ${ }^{59}$ Co nuclei in a specific local environment[13-15, $24-$ 
27].

The specific fitting parameters are as follows: The resonance frequency of each sub-line corresponds to the hyperfine field of Co within a distinct local environment. The line width of each line reflects the structural contributions not only in the first coordination shell (next nearest neighbor) but also contributions from higher coordination shells with a rule of thumb that the broader the line the more disordered local environments are. In general, the full width at half maximum (FWHM) reflects the disorder distribution as well as the information of the quadrupole splitting. In the present work the quadrupole splitting is smaller than the line width. The area of every Gaussian line correlates to the number of probed nuclei within a local environment.

The model that both describes the data best and explains the origin of all lines within a reasonable structural model for both films consists of five Gaussian lines (see Fig. 1 upper panel (a) and (b)). The resulting fit parameters (frequency, area of Gaussian line, FWHM) for both films are listed in Tab. I and Tab. II. In the following, we will discuss the origin and the meaning of each sub-line.

\section{A. Structural Model}

For the sake of clarity, all individual Gaussian lines that sum up to the total NMR spectra of the films with $10 \mathrm{~nm}$ thickness (grey line representing the total fit as a sum of individual Gaussian lines) and $25 \mathrm{~nm}$ (orange line) have their color code (see Fig.1).

Since the transition energies between $h c p$ and $f c c$ Co allotropes are small[8, 9], one may assign two sub-lines to stem from $\mathrm{Co}$ in $h c p$ and $f c c$ environment. Indeed, both spectra have lines at approximately the resonance frequency known for bulk Co, namely $\mathrm{f}\left(\mathrm{Co}_{h c p \perp}\right)=226 \mathrm{MHz}$ (hcp Co with the $c$-axis perpendicular to the magnetization direction) and $\mathrm{f}\left(\mathrm{Co}_{f c c}\right)=217 \mathrm{MHz}[24]$ but both with a small frequency shift compared to the bulk. We will come back to this later in section II C.

Similar resonance lines have previously also been reported in Co thin films [15, 23, 28]. Please note that there are two resonance lines for $h c p$ Co that differ in their orientation of the magnetization direction with respect to the crystallographic $c$ axis: $h c p_{\|}$along the $c$-axis at $220 \mathrm{MHz}$ and $h c p_{\perp}$ perpendicular to the $c$-axis at $226 \mathrm{MHz}$ [29]. The chosen experimental setup in combination with the crystallographic orientation of the Co thin films allows only 
the measurement of the $h c p_{\perp}$ signal [29]: the coil is wrapped around the sample so that the RF pulse induces pulsed magnetic fields along the in-plane direction of the film. It is justified to assign the line at $226 \mathrm{MHz}$ to $h c p_{\perp}$.

Since both Co allotropes differ in their stacking order only, it seems natural to assign the two lines between those for $\mathrm{Co}_{h c p}$ and $\mathrm{Co}_{f c c}$ as stacking fault environment (sfs) caused by the transition from metastable $f c c$ stacking induced by the lattice mismatch with the $\mathrm{Pt}$ seeding layer to the hcp stacking.

As an alternative model for the observation of the two lines between those for $\mathrm{Co}_{h c p}$ and $C_{f c c}$, Andreev et al. [30] discuss the occurrence of these resonance lines with respect to their magnetic properties and the presence of magnetic domains and domain walls as alternative interpretation. Accordingly, the resonance signals are assigned to Co within a domain or a domain wall of the $f c c$ or $h c p$ environments. This shift in frequency for $h c p$ Co within the domain and the domain wall was also reported by Malinowska et al. [31]. However, they also pointed out that long pulses and/or a much larger RF power compared to our experimental parameters are necessary to observe magnetic domain wall signals (see section IV). With this knowledge, we assign the lines between the $f c c$ and $h c p$ signal to stacking fault environments.

Accordingly, the lines at about $219 \mathrm{MHz}$ and $221 \mathrm{MHz}$ are assigned to the stacking fault environments and further denoted as sfs I and as sfs II following the work of Toth and Ravitz [15]. They calculated the resonance frequencies of the stacking faults in terms of nextnearest-planar violations concerning a pure face-centered cubic stacking order. Subsequently, sfs I corresponds to an intrinsic stacking fault $(A B C A C A B C)$ and sfs II corresponds to a stacking order with two missing planes $(A C B C B C B A)$. The assignment of those lines originating from within the Co stacking faults is in good agreement with previous literature concerning Co in bulk [14-17] and multi layers [18-21].

The fifth lines at about $203 \mathrm{MHz}$ correspond to Co nuclei situated in the grain boundaries (gb). This is due to the polycrystalline nature of the film stack and its relative contribution does not change with the thickness of the Co films. The given literature reports the occurrence of a similar grain boundary signal at about $200 \mathrm{MHz}[11,17,32]$. 


\section{B. Contribution of Each Local Structure Environment}

As explained in the previous section, we need five Gaussian lines to fit spectra of both the $10 \mathrm{~nm}$ and the $25 \mathrm{~nm}$ film with the resonance with the lowest frequency (red Gaussian line in panels (a),(b) of Fig. 1) stemming from Co nuclei at the grain boundaries, a line for $f c c$ (green) and hcp (magenta) ordering and two lines arising from Co nuclei within stacking faults (cyan and petrol). The relative contribution of each line to the overall spectrum and, hence, the relative structural contribution is extracted from the area under every Gaussian line of the fit. The resonance frequencies, the FWHM and the relative ratio of the area of the Gaussian fits for each environment are displayed in Tab. I (10 nm) and Tab. II (25 nm).

Fig. 2 shows the relative and absolute (inset) phase composition of the two thin films. It is clear that the relative phase composition change drastically with rising film thickness $t$. Specifically, we can draw several concrete conclusions based on Fig. 1 and Fig. 2:

While in the $10 \mathrm{~nm}$ thin-film $f c c$ stacking is the overall dominant phase, the majority phase will become $h c p$ for the $25 \mathrm{~nm}$ thin-film concomitantly with a strong decrease in the $f c c$ ratio. In absolute values, about $7 \mathrm{~nm}$ of $10 \mathrm{~nm}$ compared to $5 \mathrm{~nm}$ of $25 \mathrm{~nm}$ are $f c c$ type ordered (seen in the inset of Fig. 2). On the other hand, $1 \mathrm{~nm}$ of $10 \mathrm{~nm}$ is of hcp structure, and $8 \mathrm{~nm}$ out of $25 \mathrm{~nm}$ in the thicker film show hexagonal symmetry. The absolute decrease in the thickness of the $f c c$ phase between the two films may be due to the fact that two individual films were analyzed.

While the sfs I (cyan) at 219.3 MHz and sfs II (petrol) at $221 \mathrm{MHz}$ are just minority but clearly discernible contributions to the $10 \mathrm{~nm}$ thin film taking up only $4 \%$ and approximately $16 \%$ of the Co film volume, the ratios of the stacking faults rises up to $21 \%$ for the sfs I at $219 \mathrm{MHz}$ and to roughly $23 \%$ for the sfs II at $221.8 \mathrm{MHz}$ in the $25 \mathrm{~nm}$ thin film.

The line describing the Co nuclei in the grain boundaries needs to be taken into account in order to get a good fit. The relative amount of about $3 \%$ at $203 \mathrm{MHz}$ does not change with increasing film thickness, as expected assuming that the grain size does not change with increasing film thickness and the relative contribution stays constant.

We cannot locate the exact position of the different phases within the Co stacks because ZF NMR is an integral method. But the qualitative TEM study conducted in the work of Lucassen et al. supports our model[10] and allows to locate the respective environments within the film stacks. Therefore we can safely assume that we will find the $f c c$ phase at 


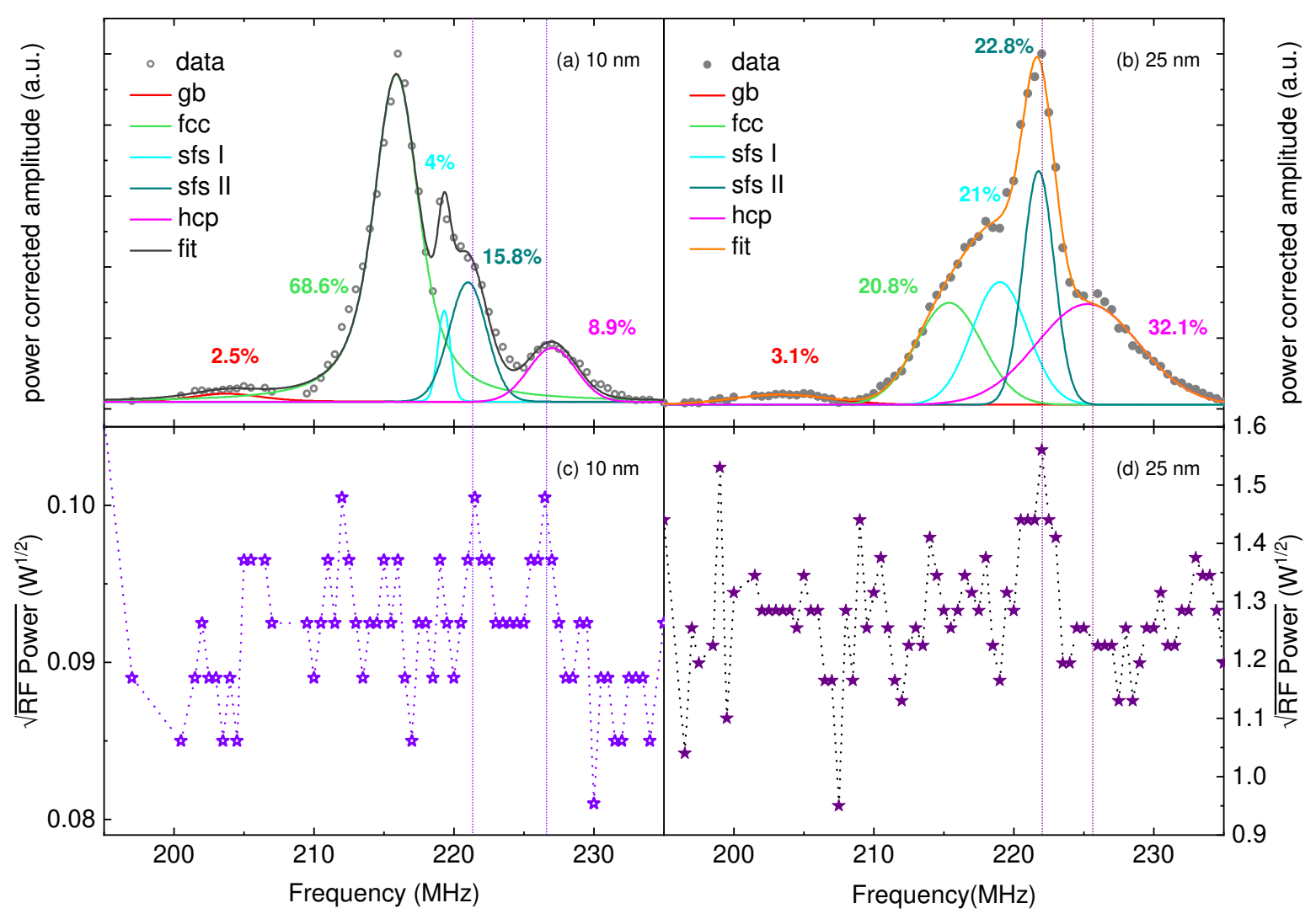

Figure 1: ${ }^{59}$ Co zero-field NMR spectra for films with the thicknesses $\mathrm{t}=10 \mathrm{~nm}(\mathrm{a})$ and $\mathrm{t}=25 \mathrm{~nm}$ (b) and their respective optimal RF power sweep (c), (d). The vertical lines crossing the top and lower panels connect distinct features in the optimal RF power with the structural contribution of the NMR spectrum (see text for details).

the bottom of the Co stack because of the lattice strain introduced by the Pt (111) layer. With increasing thickness, the influence of the Pt decreases which causes the formation of $h c p$ Co. This result is backed up by the comparable stagnant composition of the $f c c$ phase in both film stacks. The two allotropes $f c c$ and $h c p$ are not separated by a harsh boundary but are separated by stacking faults. We assume that we find a number of stacking faults in between the two ordered Co phases and we can assume to find some stacking faults within the hcp phase.

In a nutshell, the $10 \mathrm{~nm}$ thin film is mostly $f c c$ ordered, while the thicker $25 \mathrm{~nm}$ film is much more heterogeneously ordered with the largest contribution being $h c p$, closely followed by the sfs II contribution and the almost equal amounts of sfs I and $f c c$ type order. 
Table I: ${ }^{59} \mathrm{Co}$ NMR peak positions, FWHM and area ratios of the $10 \mathrm{~nm}$ film obtained from fitting with five Gaussian lines representing Co nuclei in grain boundaries (gb), face-centered cubic ( $f c c)$, hexagonal close-packed (hcp) and stacking faults (sfs I and sfs II) environments.

\begin{tabular}{|c|c|c|c|}
\hline & \multicolumn{3}{|c|}{ Frequency $[\mathrm{MHz}]$ FWHM $[\mathrm{MHz}]$ rel. ratio $(\%)$} \\
\hline $\mathrm{gb}$ & $203.8 \pm 2.5$ & $6.0 \pm 8.9$ & 2.5 \\
\hline$f c c$ & $215.9 \pm 0.5$ & $3.9 \pm 0.5$ & 68.6 \\
\hline sfs I & $219.3 \pm 0.5$ & $1.1 \pm 0.5$ & 4.0 \\
\hline sfs II & $221.0 \pm 0.6$ & $3.2 \pm 1.0$ & 15.8 \\
\hline$h c p$ & $227.0 \pm 0.5$ & $4.0 \pm 1.2$ & 8.9 \\
\hline
\end{tabular}

Table II: ${ }^{59}$ Co NMR peak positions, FWHM and area ratios of the $25 \mathrm{~nm}$ film obtained from fitting with five Gaussian lines representing Co nuclei in grain boundaries (gb), face-centered cubic $(f c c)$, hexagonal close-packed (hcp) and stacking faults (sfs I and sfs II) environments.

\begin{tabular}{r|rrc}
\hline \hline & Frequency [MHz] & FWHM [MHz] & relative ratio (\%) \\
\hline $\mathrm{gb}$ & $203.4 \pm 0.9$ & $8.3 \pm 2.5$ & 3.1 \\
$f c c$ & $215.4 \pm 2.6$ & $5.5 \pm 2.1$ & 20.8 \\
$\mathrm{sfs} I$ & $219.0 \pm 0.9$ & $4.7 \pm 3.3$ & 21.0 \\
$\mathrm{sfs}$ II & $221.8 \pm 0.5$ & $2.6 \pm 0.5$ & 22.8 \\
$h c p$ & $225.3 \pm 0.5$ & $8.6 \pm 0.8$ & 32.1 \\
\hline \hline
\end{tabular}

\section{Strain Analysis}

The lattice mismatch between the Pt layer and the adjacent $f c c$ Co layer on top of that is about $9.4 \%$ [33]. A lattice mismatch in that order of magnitude is known to cause a shift in resonance frequencies. Upon comparing the measured frequencies (see tables I and II) with reported frequencies from relaxed bulk measurements, $\mathrm{f}\left(\mathrm{Co}_{f c c}\right)=217 \mathrm{MHz}$ and $\mathrm{f}\left(\mathrm{Co}_{h c p}\right)=226 \mathrm{MHz}$ [34], we clearly recognize a frequency shift in our samples.

The measured resonance frequencies (f) are directly related to the hyperfine fields $\left(\mathrm{B}_{h f}\right)$ of the respective local environment which can be calculated with the formula

$$
f=B_{h f} * \gamma
$$




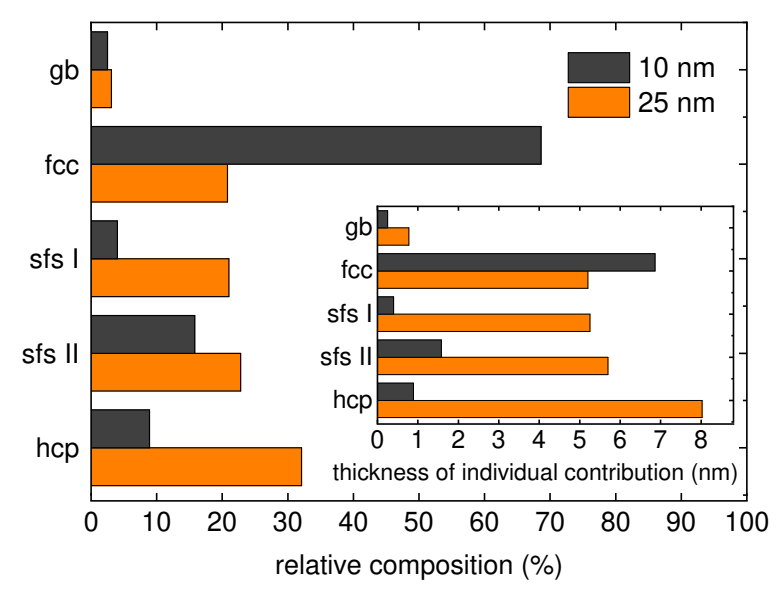

Figure 2: Relative phase composition as extracted from ${ }^{59} \mathrm{Co} \mathrm{ZF}$ NMR data for Co films with thicknesses $\mathrm{t}=10 \mathrm{~nm}$ (grey) and $\mathrm{t}=25 \mathrm{~nm}$ (orange). The inset shows the absolute phase composition in relation to the thickness of the corresponding individual structural contribution.

with the gyromagnetic ratio for Co $\gamma(\mathrm{Co})=10.054 \mathrm{MHz} / \mathrm{T}$.

The relative change in volume is calculated with the formula

$$
\frac{\Delta B_{h f}}{B_{h f}}=-1.12 \frac{\Delta V}{V}
$$

as elaborated in several previous studies specifically for Co $[19,35,36]$. The change in the cell volume is used as a measure for the strain perceived by the NMR active nuclei. The results of our strain analysis and the resulting relative volume changes of both films for the two main phases $f c c$ and $h c p$ Co are listed in Tab. III. The signs indicate whether the calculated relative volume change is compressive $(-)$ or tensile $(+)$. The values for $\frac{\Delta V}{V}$ of under $1 \%$ show that the Co thin films are indeed relaxed. This finding is in very good agreement with the study on lattice strain in Co thin films performed by Lundgren et al. [33] indicating that the Co thin films relax already after a few monolayers. The hypothetical strain of $9.4 \%$ corresponding to the lattice mismatch between $\mathrm{Pt}(111)$ and $\mathrm{Co}_{f c c}(111)$ is relaxed by formation of dislocations and grain boundaries which can be seen in the HRTEM images in the supplemental of the study conducted by Lucassen et al. [10]. Hence, the contribution of magneto-elastic anisotropy originating in strain and concomitantly its impact on the volume term of the oop magnetic anisotropy $K$ of the whole film stacks is negligible. 
Table III: The measured resonance frequencies and the corresponding hyperfine field of the thin films and the resulting change in volume compared to relaxed bulk $\mathrm{Co}, \mathrm{f}\left(\mathrm{Co}_{f c c}\right)=217 \mathrm{MHz}$ and $\mathrm{f}\left(\mathrm{Co}_{h c p}\right)=226 \mathrm{MHz}[34]$.

\begin{tabular}{r|lcc}
\hline \hline & $\nu[\mathrm{MHz}]$ & $\mathrm{B}_{h f}[\mathrm{~T}]$ & $\frac{\Delta V}{V}[\%]$ \\
\hline fcc, $10 \mathrm{~nm}$ & 215.9 & 21.47 & -0.45 \\
fcc, $25 \mathrm{~nm}$ & 215.4 & 21.42 & -0.66 \\
hcp, $10 \mathrm{~nm}$ & 227.0 & 22.58 & +0.39 \\
hcp, $25 \mathrm{~nm}$ & 225.3 & 22.41 & -0.28 \\
\hline \hline
\end{tabular}

\section{Local Magnetic Stiffness}

The optimal RF power is determined by a Gaussian fit of the RF power sweep for each frequency step. The elevated noise level in the power plot is caused by small variations in the Gaussian fit between the frequency steps, this is explained in detail in the supplemental information. A sweep for the optimal RF power at every frequency step offers insight about the magnetic stiffness of the NMR active nucleus residing in a specific local environment [13].

To further elucidate the different contributions to the magnetic anisotropy found in the volume term of the oop anisotropy $K$, we obtained the magnetic anisotropy on a local scale using the local magnetic stiffness obtained via ZF NMR. The magnetic stiffness is directly linked to the restoring field $H_{\text {rest }}$ which in turn is an effective magnetic field describing the resistance to magnetic oscillations. $H_{\text {rest }}$ is proportional to the square root of the optimal power of the applied RF pulses during the ZF NMR experiment and may be seen as the local analog compared to the macroscopic domain wall stiffness contributing to the macroscopic coercive field[37].

The lower panels in Fig. 1 display the optimal RF power at each frequency step for the $10 \mathrm{~nm}(\mathrm{c})$ and $25 \mathrm{~nm}(\mathrm{~d})$ film.

Although the RF power spectra are considerable noisy, it is a possible to discriminate between noise and signal. For that reason it is very important to carefully examine the fitting of the raw data. The details of this analysis are presented in the supplemental information of this paper. This procedure opens up new details in the optimal RF power spectra and is 
crucial for the interpretation of the given data set which will be discussed in the following.

As a first result, the overall optimal power required to achieve saturation of the spin echo intensity in the $25 \mathrm{~nm}$ film is one order of magnitude higher as in the $10 \mathrm{~nm}$ film. This may originate in the higher magnetocrystalline anisotropy in the hcp environment that is the majority contribution in the $25 \mathrm{~nm}$ film. However, this would only affect the $h c p$ environment and would not elevate the RF power for the whole film.

The pure hcp environment itseld is magnetically stiffer than the $f c c$ environment, as expected. Surprisingly, the magnetically stiffest contribution in the $25 \mathrm{~nm}$ film isst not the pure $h c p$ environment, but the Co nuclei residing in the sfs II environment of the $25 \mathrm{~nm}$ film. The vertical dotted lines crossing the top and bottom panels connect distinct features in the optimal RF power with the structural contribution of the NMR spectrum at $221 \mathrm{MHz}$ corresponding to the sfs II environment and at 225-227 MHz corresponding to the hcp environment. The rise in the overall RF power could be a result of defect pinning: Sort et al. reported on ball-milled bulk Co particles[17] where a certain concentration of stacking faults magnetically hardens the material due to pinning of the magnetic domain walls. With longer milling time, e.g., the introduction of a higher number of stacking faults, this effect is mitigated which can also be found in hexagonal Co alloys such as $\mathrm{SmCo}_{5}[17]$.

The overall increase of the magnetic stiffness within the film and the environments therein might be a product of the coupling through the iDMI between the top and bottom surfaces of the film with the stacking faults which causes the magnetic domain wall pinning.

The fact that the stacking faults, in particular the sfs II environment, have a higher magnetic stiffness than the hcp phase may indicate that the simple model used by Lucassen et al. to extract the iDMI is incomplete by assuming a hard transition between $f c c$ and hcp. We measured that there is an overall rise in the optimal RF power in dependence of the film thickness with an additional distinct peak in the sfs II environment. The optimal RF power is linked to $H_{\text {rest }}$ which has the magnetic anisotropy as a component. Consequently the rise in the measured $\mathrm{RF}$ power is connected to a rise in the magnetic oop anisotropy $K$. The rise in $h c p$ and the stacking faults and their quantification by ZF NMR could help to analyze the magnetic anisotropies and non-reciprocities for samples with thickness above $25 \mathrm{~nm}$ in the future. 


\section{SUMMARY}

In this work, we showed how the different local Co environments evolve with rising thickness in Co nanofilm stacks with Pt (111) as a seeding and Ir as a capping layer. We investigated the local structure and local magnetic stiffness and their contribution to the oop anisotropy $K$. Using ZF NMR, we showed that the absolute phase contribution changes drastically for the hcp and stacking faults (sfs) environments but remains stagnant for the $f c c$ environment if we compare the $10 \mathrm{~nm}$ stack with the $25 \mathrm{~nm}$. We concluded that the change in the overall oop magnetic anisotropy is not strain-driven due to lattice mismatch. Analyzing the optimal RF power extracted from the ZF NMR data as a measure of the magnetic stiffness we can make some qualitative statements about the oop magnetic anisotropy $K$

This analysis shows us a distinct increase in the overall optimal RF power of one order of magnitude between the two examined film stack which is linked to the oop magnetic anisotropy $K$.

\section{METHODS}

\section{A. Thin film preparation}

The film samples were grown using parallel face-to-face target and substrate $\operatorname{Ar} D C$ magnetron sputter deposition from 2 inch targets in a system with a base pressure of $\approx$ $3 \times 10^{-8}$ mbar. The studied samples consist of $(\mathrm{Ta}(4 \mathrm{~nm}) / \operatorname{Pt}(4 \mathrm{~nm}) / \mathrm{Co}(t) / \operatorname{Ir}(3 \mathrm{~nm}) / \operatorname{Pt}(2 \mathrm{~nm})$ on a $100 \mathrm{~nm} \mathrm{SiO}_{2}$ coated Si substrate with thicknesses $t=10 \mathrm{~nm}<t_{c r}<t=25 \mathrm{~nm}$. For details of the film preparation, we refer to previous work (see [38]).

\section{B. ${ }^{59}$ Co nuclear magnetic resonance spectroscopy (NMR)}

The ${ }^{59} \mathrm{Co}$ NMR measurements were performed at a temperature of $5 \mathrm{~K}$ in an automated, coherent, phase-sensitive, and frequency-tuned spin-echo spectrometer (NMR Service, Erfurt, Germany). In order to increase the signal intensity that scales with the number of probed ${ }^{59} \mathrm{Co}$ nuclei, the films were cut in two parts, the resulting two slices were sandwiched with the substrate side facing each other. All resonance spectra were recorded over a fre- 
quency range of $190-240 \mathrm{MHz}$ with a step size of $0.5 \mathrm{MHz}$, with a $90^{\circ}-90^{\circ}$ pulse of $0.6 \mu \mathrm{s}$ pulse length and a $\mathrm{T}_{1}$ of $100 \mathrm{~ms}$. The recorded ${ }^{59} \mathrm{Co}_{\mathrm{NMR}}$ spectra were corrected for the enhancement factor (see, e.g., references $[27,34,39]$ ) and for the $\nu^{2}$-dependence. All spectra were normalized for their respective most prominent resonance line to allow for comparison. The spectra were fitted with an appropriate Gaussian model. The area of each Gaussian curve corresponds to the relative number of $C o$ in the respective environment. The optimal RF power was extracted from the power-correction procedure as introduced, e.g., in references $[34,39]$.

\section{ACKNOWLEDGMENT}

Financial support is acknowledged from the Deutsche Forschungsgemeinschaft (DFG) through Grants No. WU595/3-3 and No.WU595/14-1 and the Sonderforschungsbereich SFB 1143 (B01). This work is part of the research program of the Foundation for Fundamental Research on Matter (FOM), which is part of the Netherlands Organization for Scientific Research (NWO). This work is also supported in part by the European Research Council via Consolidator Grant number 725509 SPINBEYOND.

\section{AUTHOR CONTRIBUTION}

PF conducted the ZF NMR measurement, PF and SW analyzed and interpreted the NMR data. JL was responsible for the fabrication of the film samples, and together with HJMS and RL instigated the original study into the thin film Co crystal structure. PF, SW, JL and RL wrote the manuscript. All authors contributed to the discussion of the results and reviewed the manuscript. 


\section{COMPETING INTERESTS}

The author(s) declare no competing interests.

[1] I. Dzyaloshinsky, "A thermodynamic theory of "weak" ferromagnetism of antiferromagnetics," Journal of Physics and Chemistry of Solids, vol. 4, pp. 241-255, jan 1958.

[2] T. Moriya, "Anisotropic superexchange interaction and weak ferromagnetism," Physical Review, vol. 120, pp. 91-98, oct 1960 .

[3] A. Thiaville, S. Rohart, E. Jue, V. Cros, and A. Fert, "Dynamics of dzyaloshinskii domain walls in ultrathin magnetic films," EPL (Europhysics Letters), vol. 100, p. 57002, dec 2012.

[4] N. Grisewood, J. Eves, T. Usher, and H.-B. Braun, "Chiral magnetization configurations in magnetic nanostructures in the presence of dzyaloshinskii-moriya interactions," Journal of Applied Physics, vol. 111, p. 07C706, apr 2012.

[5] J.-H. Moon, S.-M. Seo, K.-J. Lee, K.-W. Kim, J. Ryu, H.-W. Lee, R. D. McMichael, and M. D. Stiles, "Spin-wave propagation in the presence of interfacial dzyaloshinskii-moriya interaction," Physical Review B, vol. 88, nov 2013.

[6] J. Cho, N.-H. Kim, S. Lee, J.-S. Kim, R. Lavrijsen, A. Solignac, Y. Yin, D.-S. Han, N. J. J. van Hoof, H. J. M. Swagten, B. Koopmans, and C.-Y. You, "Thickness dependence of the interfacial dzyaloshinskii-moriya interaction in inversion symmetry broken systems," Nature Communications, vol. 6, jul 2015.

[7] S. Tacchi, R. Troncoso, M. Ahlberg, G. Gubbiotti, M. Madami, J. Åkerman, and P. Landeros, "Interfacial dzyaloshinskii-moriya interaction in pt/CoFeB films: Effect of the heavy-metal thickness," Physical Review Letters, vol. 118, apr 2017.

[8] P. Toledano, G. Krexner, M. Prem, H.-P. Weber, and V. Dmitriev, "Theory of the martensitic transformation in cobalt," Physical Review B, vol. 64, sep 2001.

[9] M. T. Johnson, P. J. H. Bloemen, F. J. A. den Broeder, and J. J. de Vries, "Magnetic anisotropy in metallic multilayers," Reports on Progress in Physics, vol. 59, pp. 1409-1458, nov 1996.

[10] J. Lucassen, C. F. Schippers, M. A. Verheijen, P. Fritsch, E. J. Geluk, B. Barcones, R. A. Duine, S. Wurmehl, H. J. M. Swagten, B. Koopmans, and R. Lavrijsen, "Extraction of 
dzyaloshinskii-moriya interaction from propagating spin waves," Physical Review B, vol. 101, feb 2020 .

[11] P. Panissod, C. Mèny, M. Wójcik, and E. Jedryka, "Magnetic properties and structure of metallic multilayers investigated by NMR," MRS Proceedings, vol. 475, 1997.

[12] M. Malinowska, M. Wojcik, S. Nadolski, E. Jedryka, C. Meny, P. Panissod, M. Knobell, A. Viegas, and J. Schmidt, "Identification of magnetic phases in granular co10cu90 alloy using NMR method," Journal of Magnetism and Magnetic Materials, vol. 198-199, pp. 599-601, jun 1999.

[13] M. C. Panissod, P., "Nuclear Magnetic Resonance Investigations of the Structure and Magnetic Properties of Metallic Multilayers and Nanoncomposites," Applied Magnetic Resonance, vol. 19, pp. 447-460, 2000.

[14] A. C. Gossard and A. M. Portis, "Observation of nuclear resonance in a ferromagnet," Physical Review Letters, vol. 3, pp. 164-166, aug 1959.

[15] L. E. Toth and S. F. Ravitz, "Ferromagnetic nuclear resonance in cobalt nuclei in stacking faults and twins," Journal of Physics and Chemistry of Solids, vol. 24, no. 10, pp. 1203-1206, 1963.

[16] D. C. Creagh, S. G. Bailey, and G. V. H. Wilson, "X-ray and NMR investigations of cobalt," Philosophical Magazine, vol. 32, pp. 405-415, aug 1975.

[17] J. Sort, S. Suriñach, J. S. Muñoz, M. D. Baró, M. Wojcik, E. Jedryka, S. Nadolski, N. Sheludko, and J. Nogués, "Role of stacking faults in the structural and magnetic properties of ball-milled cobalt," Physical Review B, vol. 68, jul 2003.

[18] R. Allenspach, M. Stampanoni, and A. Bischof, "Magnetic domains in thin epitaxial co/au (111) films," Physical Review Letters, vol. 65, no. 26, p. 3344, 1990.

[19] E. A. M. van Alphen, "Nuclear magnetic resonance studies of co-based multilayers," 1995.

[20] K. L. Dang, P. Veillet, H. He, F. J. Lamelas, C. H. Lee, and R. Clarke, "NMR study of interface structure in epitaxial co-cu superlattices," Physical Review B, vol. 41, pp. 12902-12904, jun 1990.

[21] H. de Gronckel, J. Bienert, F. den Broeder, and W. de Jonge, "Co/x multilayers, NMR study of microscopic structure and strain," Journal of Magnetism and Magnetic Materials, vol. 93, pp. 457-461, feb 1991.

[22] M. Cerisier, K. Attenborough, E. Jedryka, M. Wojcik, S. Nadolski, C. V. Haesendonck, and 
J. P. Celis, "Structural study of nanometric electrodeposited co films using 59co NMR," Journal of Applied Physics, vol. 89, pp. 7083-7085, jun 2001.

[23] H. A. M. de Gronckel, P. J. H. Bloemen, E. A. M. van Alphen, and W. J. M. de Jonge, "Structure and local magnetic anisotropy of MBE-grown co films," Physical Review B, vol. 49, pp. 11327-11335, apr 1994.

[24] G. G. Strijkers, "Magnetic nanostructures : an experimental study of structural, magnetic and transport properties," 1999.

[25] C. Meny, P. Panissod, and R. Loloee, "Structural study of cobalt-copper multilayers by NMR," Physical Review B, vol. 45, pp. 12269-12277, jun 1992.

[26] J. P. Jay, M. Wojcik, and P. Panissod, "Hyperfine field and ordering in bcc CoFe bulk alloys studied by 59co NMR and monte-carlo simulation," Zeitschrift für Physik B Condensed Matter, vol. 101, pp. 471-486, dec 1996.

[27] S. Wurmehl and J. T. Kohlhepp Journal of Physics D: Applied Physics, vol. 41, p. 173002, 2008 .

[28] D. Fekete, H. Boasson, A. Grayevski, V. Zevin, and N. Kaplan, "Anisotropic hyperfine interactions in ferromagnetic hcp co," Phys. Rev. B, vol. 17, pp. 347-354, Jan 1978.

[29] G. J. Strijkers, J. T. Kohlhepp, H. J. M. Swagten, and W. J. M. de Jonge, "Nuclear magnetic resonance study of epitaxial co layers on single-crystal substrates," Applied Magnetic Resonance, vol. 19, pp. 461-469, 2000.

[30] A. S. Andreev, O. B. Lapina, and S. V. Cherepanova, "A new insight into cobalt metal powder internal field 59co NMR spectra," Applied Magnetic Resonance, vol. 45, pp. 1009-1017, aug 2014

[31] M. Malinowska, C. Meny, E. Jedryka, and P. Panissod, "The anisotropic first-neighbour contribution to the hyperfine field in hexagonal-close-packed co: a nuclear magnetic resonance study of diluted alloys and multilayers," Journal of Physics: Condensed Matter, vol. 10, pp. 4919-4928, jun 1998.

[32] K. L. Dang, P. Veillet, E. Vélu, S. S. P. Parkin, and C. Chappert, "Influence of crystal structure on the magnetoresistance of co/cu multilayers," Applied Physics Letters, vol. 63, pp. 108-110, jul 1993.

[33] E. Lundgren, B. Stanka, M. Schmid, and P. Varga, "Thin films of co on pt(111): Strain relaxation and growth," Physical Review B, vol. 62, pp. 2843-2851, jul 2000. 
[34] P. C. Riedi, T. Thomson, and G. J. Tomka, "Nmr of thin magnetic films and superlattices," in Handbook of Magnetic Materials (K. H. J. Buschow, ed.), vol. 12, p. 97, Amsterdam, London, New York, Tokyo: North-Holland, 1999.

[35] R. Jones and I. Kaminow, "Volume dependence of the nmr in ferromagnetic cobalt," Bull. Am. Phys. Soc, vol. 5, p. 175, 1960.

[36] J. Janak, "Calculated hyperfine fields and their pressure derivatives in fe, co, and ni," Physical Review B, vol. 20, no. 5, p. 2206, 1979.

[37] M. Belesi, L. Giebeler, C. G. F. Blum, U. K. Rößler, B. Büchner, and S. Wurmehl, "Local magnetism and structural properties of heusler ni ${ }_{2}$ MnGa alloys," Phys. Rev. B, vol. 91, p. 134415, Apr 2015.

[38] J. Lucassen, C. F. Schippers, L. Rutten, R. A. Duine, H. J. M. Swagten, B. Koopmans, and R. Lavrijsen, "Optimizing propagating spin wave spectroscopy," Applied Physics Letters, vol. 115, p. 012403, jul 2019.

[39] P. Panissod, "Structural and magnetic investigations by nmr. application to magnetic multilayers," in NATO ASI series High Tech (V. G. Baryakhtar, P. E. Wigen, and N. A. Lesnik, eds.), vol. 48, p. 225, Dordrecht: Kluwer Academic, 1997. 
Figures

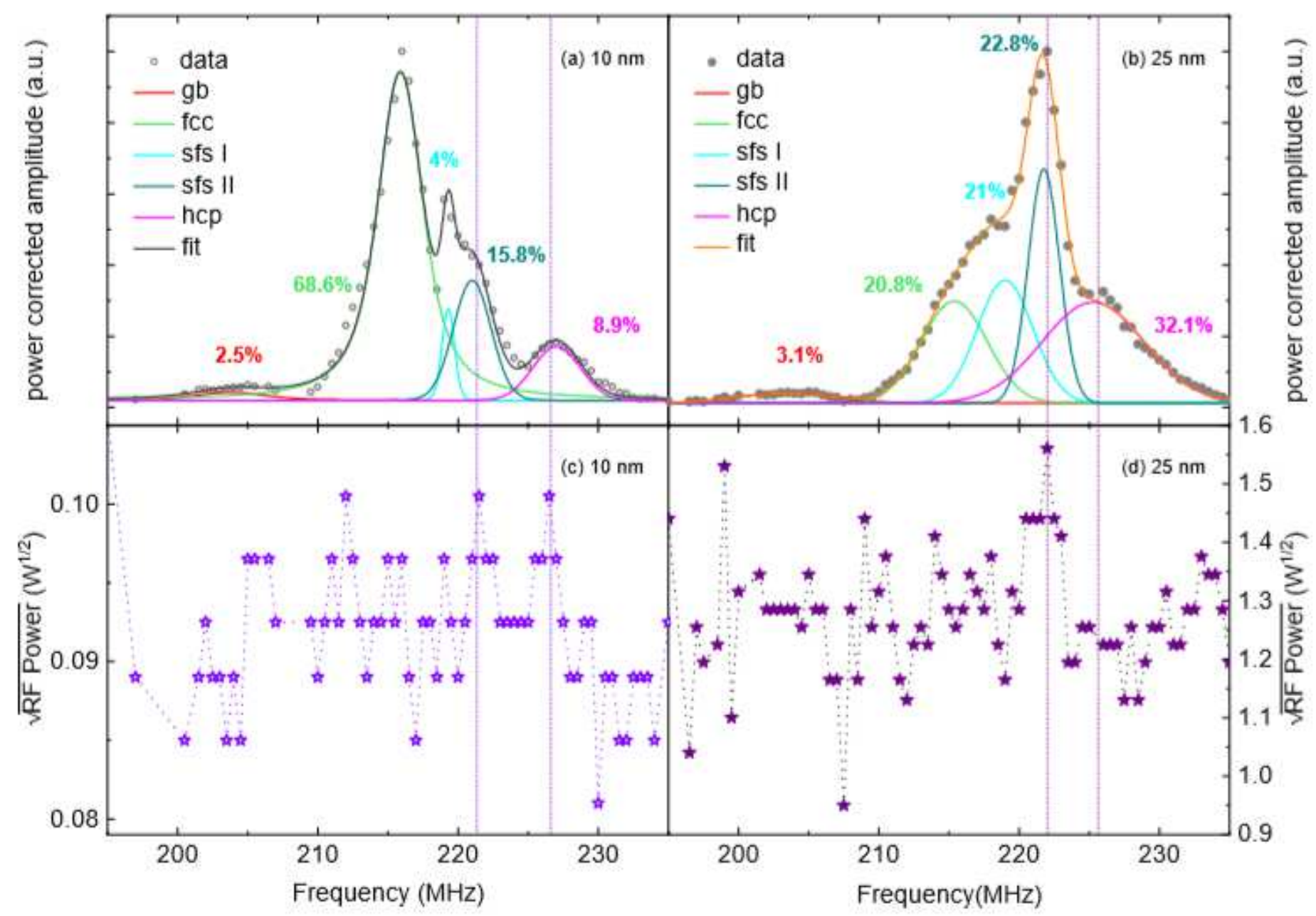

Figure 1

Co zero-field NMR spectra for films with the thicknesses $t=10 \mathrm{~nm}$ (a) and $\mathrm{t}=25 \mathrm{~nm}$ (b) and their respective optimal RF power sweep (c), (d). The vertical lines crossing the top and lower panels connect distinct features in the optimal RF power with the structural contribution of the NMR spectrum (see text for details). 


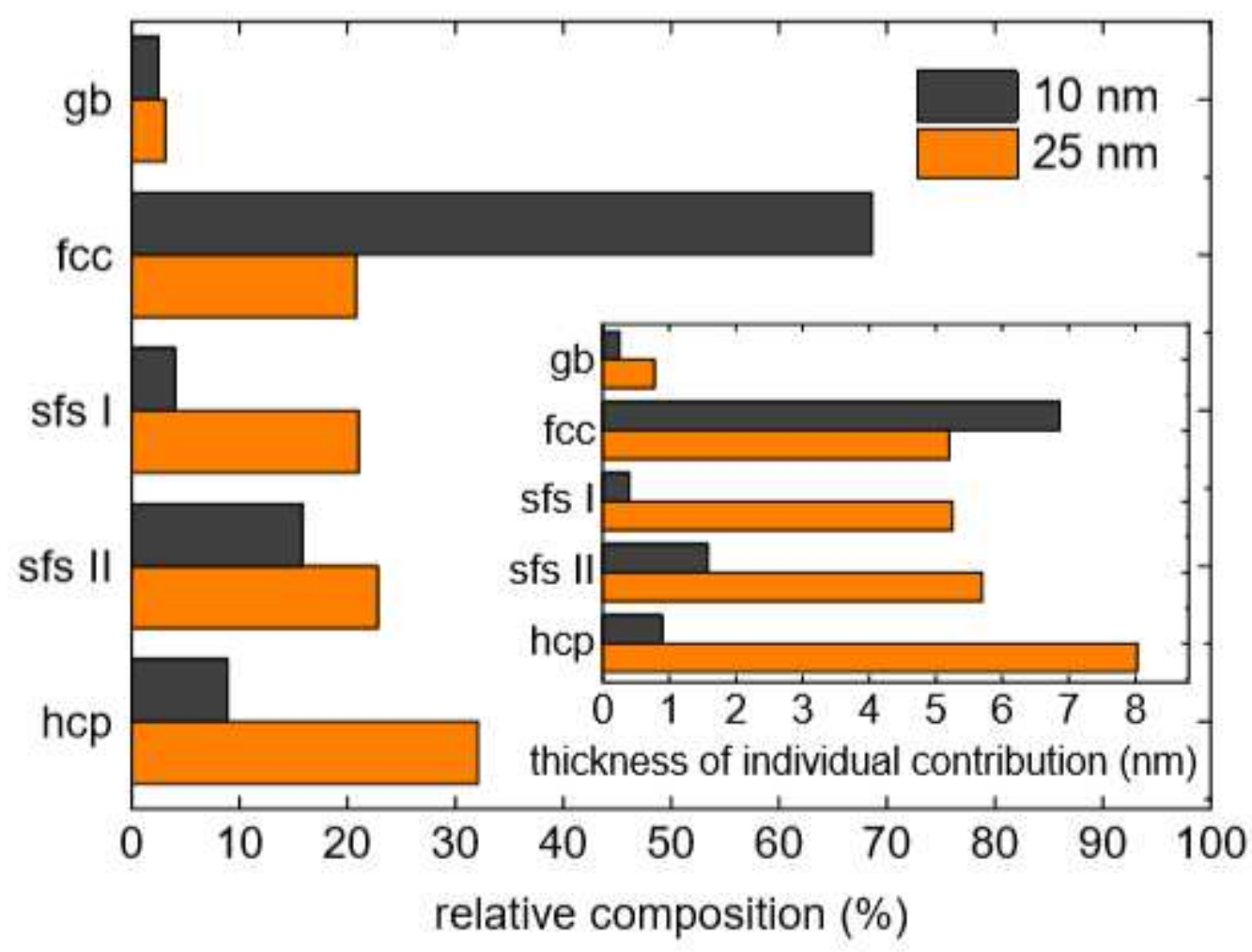

Figure 2

Relative phase composition as extracted from 59Co ZF NMR data for Co films with thicknesses $\mathrm{t}=10 \mathrm{~nm}$ (grey) and $\mathrm{t}=25 \mathrm{~nm}$ (orange). The inset shows the absolute phase composition in relation to the thickness of the corresponding individual structural contribution.

\section{Supplementary Files}

This is a list of supplementary files associated with this preprint. Click to download.

- supplementalCo.pdf 